\title{
Agilität im Klinikeinkauf: ein theoretisches Konzept oder schon gelebte Praxis?
}

\author{
Heutzutage sollen im Arbeitsleben alle möglichst flexibel im Sinne von anpassungsfähig sein - und der \\ Trend setzt sich fort. Das Schlagwort lautet daher Agilität, womit nicht weniger gemeint ist als Anpassungs- \\ fähigkeit auf höchstmöglichem Niveau. Agilität wird zum zentralen Treiber für Wettbewerbsfähigkeit. \\ Für den Praktiker erscheint es einerseits erstrebenswert, agil zu sein, andererseits wirken viele Fachbegriffe \\ wie „Scrum“ oder „Sprint“ sperrig und sind Neuland für den Klinikbereich.
}

Die allgegenwärtige Dynamik der wirtschaftlichen Rahmenbedingungen wird heute gern mit dem Kürzel VUCA umschrieben:

- V steht für Volatilität,

- U für Unsicherheit,

- C für Komplexität und

- A für Ambiguität, also die Mehrdeutigkeit von Umweltentwicklungen.

Der Beginn des 21. Jahrhunderts ist von extremen Veränderungen gekennzeichnet: Die USA geben schrittweise die Rolle der Ordnungsmacht auf, China und Russland drängen machtpolitisch nach vorne, populistische Tendenzen spalten ganze Gesellschaften und Europa als Staatenbund. Für Unternehmen kommen mit den Themen digitale Transformation, Nachhaltigkeit und Neuausrichtung der Globalisierung weitere zentrale Herausforderungen hinzu. Wer sich in diesem „Chaos“ behaupten will, muss anpassungsfähig sein.

Bis vor einigen Jahren wurde dies unter dem Begriff der Flexibilität als wesentliche betriebswirtschaftliche Herausforderung für erfolgreiche Unternehmen diskutiert. Heute lautet die große Überschrift Agilität. Tatsächlich will Agilität mehr sein als die traditionelle Flexibilität: Flexibilität ist die Fähigkeit einer Organisation, sich an unsichere aber im Wesentlichen vorhersehbare Veränderungen der Umwelt anzupassen. Agilität fordert Anpassungsfähigkeit auch an unbekannte und nicht vorhersehbare Entwicklungen. Dies wirkt auf den ersten Blick fast widersprüchlich, betont aber sehr gut, dass es um etwas Besonderes und Neues geht.

Wer nun hinterfragt, wie ein Unternehmen insgesamt und eine zentrale Funktion wie der Einkauf denn agil werden können, stößt zumeist auf Praxisberichte aus der Software-Industrie. Diese sind einerseits ganz unterhaltsam zu lesen, weil man sich gut vorstellen kann, wie kleine, schlagkräftige Teams (sogenannte Scrums) in zeitlich überschaubaren Arbeitsphasen (Sprints)

Auslöser für „hektische Betriebsamkeit“

Die Autoren haben in den vergangenen Monaten diverse Workshops mit Krankenhauseinkäufern durchgeführt und dabei auf die Frage nach Ursachen für Turbulenzen folgende typische Antworten erhalten: > Lieferanten-Insolvenz

Lieferengpässe

Produktrückrufe

Neue Hygienerichtlinie

Systemausfall

Ausfall der Logistik

$\checkmark$ Chefarzt-Wechsel

- Havarie an medizinischen Geräten

Preiserhöhungen trotz Rahmenvertrag

$\checkmark$ Epidemien und vielfältigen Rückkopplungsschleifen parallel zur Weiterentwicklung der Produktspezifikationen am Programmcode arbeiten. Andererseits erscheint die direkte Übertragbarkeit organisatorischer Konzepte von der Softwareerstellung auf andere Bereiche aber problematisch. Es fehlt schlicht an konkreten Umsetzungskonzepten.

\section{Turbulenzen analysieren}

Ein guter Startpunkt auf dem Weg zu Agilität besteht darin, zu hinterfragen, wann und wodurch in einem Tätigkeitsbereich hektische Betriebsamkeit abseits der täglichen Routine entsteht. Jeder Praktiker kennt diese Situationen: Eine externe Nachricht erfordert schnelle und spezifische Maßnahmen. Oftmals kann man die Krise meistern, aber am Ende fragt man sich schon, ob das nicht hätte geschmeidiger laufen können.

Auf die Frage nach den Ursachen für die Turbulenzen werden unter anderem auch Schwierigkeiten mit oder auf Seiten der Lieferanten genannt, zum Beispiel Lieferanten-Insolvenz, Lieferengpässe, Produktrückrufe.

Wenn das Unternehmen wüsste, was das Unternehmen schon weiß ...

Der wohl einfachste Zugang zur Lösung der Probleme besteht schlicht darin, es von den Leuten erledigen zu lassen, die das schon einmal gemacht haben. Insbesondere in Krankenhauskonzernen und großen Einkaufsverbünden ist die 


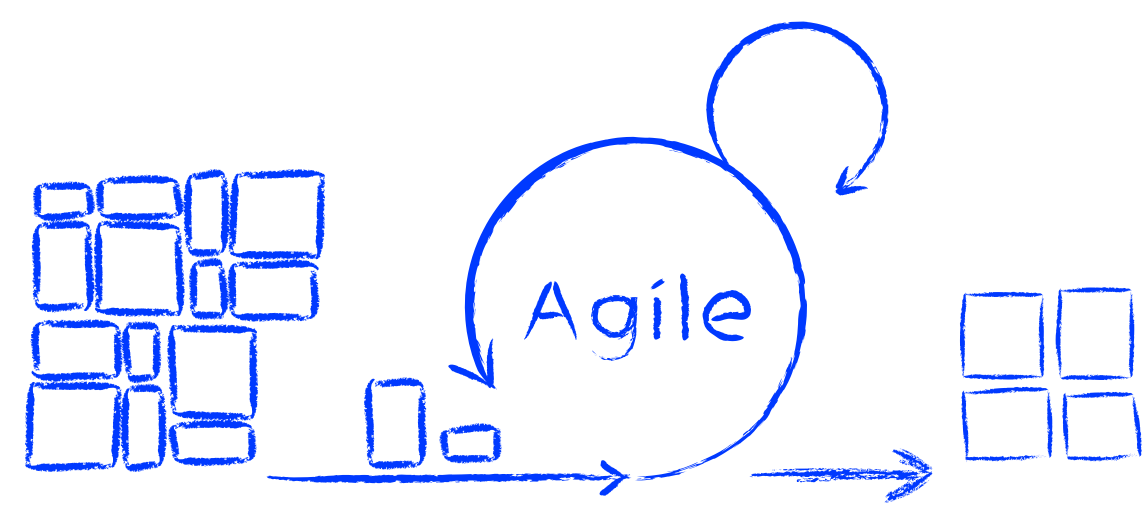

Im Krankenhaus-Einkauf gehört das Thema Agilität definitiv auf die Agenda, denn Agilität könnte zum zentralen Treiber für die Wettbewerbsfähigkeit werden. Symbolfoto: AdobeStock/Asha Sreenivas

Wahrscheinlichkeit groß, dass es vergleichbare Situationen schon zuvor gegeben hat und das Know-how zur Lösung bereits existiert. Unter dem Zeitdruck einer aktuellen Krise findet nur niemand die Zeit, sich darauf zu besinnen.

Ein großer deutscher Industriekonzern hat vor einigen Monaten ein praktikables Konzept entwickelt, wie mithilfe einer Online-Plattform sowohl die Suche nach Experten für spezielle Krisensituationen professionalisiert als auch ein geordneter Personaleinsatz vorbereitet werden kann. In einem ersten Schritt wurde eine Datenbank angelegt, in die jeder Einkäufer auf freiwilliger Basis seine speziellen Kompetenzen und Berufserfahrungen einträgt und auch kontinuierlich aktualisiert. Im Mittelpunkt stehen dabei naturgemäß genau die Fähigkeiten und Fertigkeiten, die in den oben genannten Krisenfällen benötigt werden (z. B. juristische Kenntnisse für Insolvenzen bei ausländischen Lieferanten oder besondere Markt- und Produktkenntnisse in hygienerelevanten Warengruppen).

\section{Die hohe Kunst des agilen Personal- einsatzes}

Der Identifikation von einem oder mehreren Spezialisten muss aber ein zweiter, mindestens genauso wichtiger Schritt folgen. Das Hinzuziehen von Experten aus anderen Abteilungen oder Häusern gelingt nur dann, wenn sowohl die Fachkräfte selbst als auch deren Vorgesetzte einen Vorteil darin sehen. Beides ist keine Selbstverständlichkeit. Wenn besonders qualifizierten Kräften permanent Mehrarbeit aufgelastet wird, ohne dass diese kurz- oder langfristig einen Vorteil darin sehen, wird deren Engagement eventuell nachlassen. Aus der Perspektive der entsendenden Abteilung können sich ebenfalls Probleme ergeben, weil dort möglicherweise Kapazitätslücken entstehen, die budgetär auszugleichen sind.

In dem genannten Projekt wurde dieses Spannungsfeld überwunden, indem drei Kategorien von Mitarbeiterinnen und Mitarbeitern gebildet wurden. Zum einen wurde eine Task Force eingerichtet, deren Mitglieder ausschließlich für solche Feuerwehreinsätze zuständig sind. Dies ist aber nur dann erforderlich, wenn es wirklich regelmäßige Krisensituationen gibt. Eine zweite Gruppe besteht aus solchen Fachkräften, die sich auf freiwilliger Basis für temporäre Sonderaufgaben bereitfinden und sich als potenzielle Kandidaten in die Datenbank eingetragen haben. Die dritte und entscheidende Kategorie quasi eine Ebene zwischen den beiden erstgenannten - bilden Experten, die sich nicht nur selbst als Helfer für besondere Aufgaben eingetragen haben, sondern auch von ihren direkten Vorgesetzten dazu autorisiert wurden. Dies umfasst zum einen die Bestätigung der Kompetenzen und zum anderen die Bereitschaft der entsendenden Institution, auf dem Wege einer Budgetverrechnung diese Experten auch zur Verfügung stellen zu wollen.

\section{In Gesamtzusammenhängen denken und schrittweise vorangehen}

Auch wenn es im Krankenhaus-Einkauf derzeit (noch) nicht die disruptiven Umwälzungen wie in anderen Branchen gibt, gehört Agilität auf die Agenda. Ausgangspunkt sollte stets eine qualifizierte Bestandsaufnahme sein, welche krisenhaften Situationen es bisher schon gegeben hat und was zukünftig passieren könnte. Dann kann ein gezieltes Wissensmanagement bei der Prävention und Krisenbewältigung helfen. Wenn erst einmal das richtige Personal gefunden und in ein Projektmanagement eingesteuert ist, können sukzessive weitere Schritte, zum Beispiel die Einführung digitaler Technologien auf dem langen Weg zur Agilität eingeleitet werden.

Univ.-Prof. Dr. Jörg Schlüchtermann, Inhaber des Lehrstuhls BWLV: Produktionswirtschaft und Industriebetriebslehre, Universität Bayreuth; Mitglied im Redaktionsbeirat Klinik Einkauf

Franziska Bächer, Wissenschaftliche Mitarbeiterin am Lehrstuhl BWLV: Produktionswirtschaft und Industriebetriebslehre, Universität Bayreuth

Mathias Köhler, Wissenschaftlicher Mitarbeiter am Lehrstuhl BWLV: Produktionswirtschaft und Industriebetriebslehre, Universität Bayreuth 